\title{
Characterizing cell fate
}

Chen et al. have identified and catalogued genes, transcripts and alternative splicing events that are specific to various human haematopoietic cell fates, providing insights into the transcriptional changes involved in the various haematopoeitic precursor populations.

The authors used RNA sequencing to identify cell fate-specific changes in eight primary human haematopoietic populations representing different myeloid commitment stages of haematopoiesis. Cell type expression changes were found in 6,711 genes and 10,724 transcripts, as well as in 2,301 differentially used alternative splicing events. Of note, an enrichment of non-protein-encoding transcripts during the early stages of differentiation was identified, and changes in transcripts did not always imply changes at the gene level.

They identified 29,736 splice junctions that were not annotated in Ensembl. In addition, they found cell type-specific use of alternative splice junctions that resulted in inclusion or exclusion of protein functional domains. For example, they identified an alternative splice junction used by nuclear factor I/B (NFIB), a protein that had previously been implicated in regulating lung and nervous system development. The novel NFIB transcript (NFIB-S) is highly expressed in megakaryocytes and is shorter than the canonical isoform. Contrary to the canonical isoform, NFIB-S cannot interact with its binding partner NFIC. Overexpression of NFIB-S or NFIC, but not of canonical NFIB, stimulates megakaryocyte maturation, indicating that the shorter isoform is required for this process.

The catalogue provided in this study will be central for further understanding mutations that lead to blood disorders and leukaemias.

Isabel Lokody

ORIGINAL RESEARCH PAPER Chen, L. et al. Transcriptional diversity during lineage commitment of human blood progenitors. Science 345,1251033 (2014)

\section{central for}

further

understanding

mutations that

lead to blood

disorders

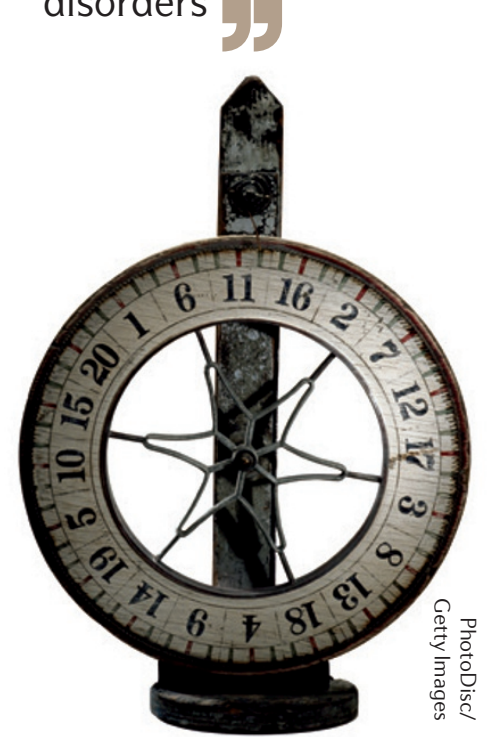

